\title{
Vitez entre les langues. De Phèdre (1975) à Anacaona (1988)
}

Vitez between Languages: From Phèdre (1975) to Anacaona (1988)

\section{Brigitte Joinnault}

\section{(2) OpenEdition}

\section{Journals}

Édition électronique

URL : https://journals.openedition.org/rsl/2547

DOI : $10.4000 /$ rsl.2547

ISSN : 2271-6246

Éditeur

Éditions Rue d'Ulm

\section{Référence électronique}

Brigitte Joinnault, «Vitez entre les langues. De Phèdre (1975) à Anacaona (1988) », Revue Sciences/ Lettres [En ligne], 6 | 2019, mis en ligne le 10 décembre 2018, consulté le 30 juillet 2021. URL : http:// journals.openedition.org/rsl/2547 ; DOI : https://doi.org/10.4000/rsl.2547

Ce document a été généré automatiquement le 30 juillet 2021

(c) Revue Sciences/Lettres 


\title{
Vitez entre les langues. De Phèdre (1975) à Anacaona (1988)
}

\author{
Vitez between Languages: From Phèdre (1975) to Anacaona (1988)
}

\author{
Brigitte Joinnault
}

Il y a des gens au monde dont la généalogie est de langage et point de sang ${ }^{1}$.

1 La traduction pour Antoine Vitez était à la fois une pratique mais aussi une éthique : « Si nous ne traduisons pas, si nous ne représentons pas, nous renonçons à la civilisation, à la vie ${ }^{2}$ ». "L'art de la traduction est pour moi une activité tout à fait analogue à mon activité de théâtre ${ }^{3}$ ", expliquait-il à Émile Copfermann dans leurs entretiens de 1981. Traduire et représenter, autrement dit mettre en scène le verbe, l'homme et leurs relations, "c'est le même travail, c'est l'art du choix dans la hiérarchie des signes ${ }^{4}$ ».

2 Georges Banu et Danièle Sallenave, dans l'anthologie qu'ils réalisèrent en 1991, parlent de "traduction généralisée » pour nommer « la passion rectrice de la vie et de l'œuvre d'un homme qui aimait la raison et croyait à son universalité ${ }^{»}$. Leur postface commence ainsi :

Au cœur de toutes les expériences qui ont formé chez Antoine Vitez le metteur en scène qu'il a été, il y a le goût, la pratique, la passion des langues. Cette passion traverse tout ; elle infuse tout son art ; elle l'a rendu poète et traducteur; elle lui a donné le goût des voix et des acteurs étrangers : en un mot, c'est la passion des langues qui a dominé sa vie, et qui a placé tout son art sous le signe de la traduction. Traduire n'était pas pour lui seulement un plaisir, une aptitude (chez lui elle était immense), une activité (il s'y consacrait fréquemment). C'était une méthode, un mode de la pensée, une philosophie. Celui pour qui traduire est l'axe de sa vie, pour celui-là tout est langue : les langues, les voix, les corps, l'histoire, la politique, le théâtre ${ }^{6}$.

3 La métaphore de la traduction, tout en soulignant l'attention unique que l'homme de théâtre portait à la langue et au rapport entre les langues, nous offre une clé de compréhension de ce que l'on pourrait appeler une philosophie de la relation et une 
manière de défendre et de mettre en scène, concrètement, grâce au théâtre, un idéal relationnel.

Si nous revenons aujourd'hui sur cet aspect fondamental de l'œuvre d'Antoine Vitez, aspect que lui-même, ses compagnons de route et les grands témoins de son travail ont déjà mis en évidence ${ }^{7}$, c'est pour donner idée de ce que la consultation des archives sonores peut apporter à l'exercice de l'appréhension d'un savoir qui ne cesse d'être surprenant, et pour montrer qu'en matières de langue et de théatre, l'écoute donne chair à l'idée, permet de l'éprouver concrètement, sensiblement, et d'en être bouleversé, sans toutefois effacer l'éloignement et sans créer l'illusion de tout comprendre, ce qui dans un cas comme celui de Vitez est essentiel, car on sait bien que l'éthique de la traduction, dans son théâtre, ne s'incarnait pas seulement sur le plan sonore mais participait d'une épaisseur théâtrale qu'aucun type d'archive pris isolément ne pourrait, à lui seul, permettre d'appréhender.

Le cas « Métellus-Vitez » occupe une place centrale dans cette étude pour des raisons multiples. Sur le plan esthétique, la question de la langue et du rapport entre les langues est un enjeu poétique et politique central dans l'œuvre du poète, linguiste et neurologue Jean Métellus, et sa pièce Anacaona offre un point d'appui remarquable pour penser le rôle de la parole dans l'histoire et dans l'actualité politique et sociale. Sur le plan historique, il nous semble juste, d'une part, de mentionner que, de 1982 à 1988, à trois reprises, des textes de Jean Métellus ont été présentés à Chaillot - présentations qui ont précédé la lecture du Discours sur le colonialisme d'Aimé Césaire au Festival d'Avignon (dans le cadre des célébrations du bicentenaire de 1789), puis la programmation de La Tragédie du roi Christophe à la Comédie-Française (en 1991) -, d'autre part, de porter attention à cette mise en scène d'Anacaona, jusqu'ici systématiquement oubliée, en partie probablement à cause du grand succès de la mise en scène de l'intégrale du Soulier de satin de Paul Claudel, également présentée dans la grande salle lors de la dernière saison d'Antoine Vitez à Chaillot, et du phénomène d'ombre portée que produisent de tels succès sur des réalisations que l'on peut considérer comme voisines ${ }^{8}$. Sur le plan méthodologique, enfin, l'étude de ce cas nous semble illustrer de façon claire l'intérêt qu'il peut y avoir à associer différentes archives sonores (quatre enregistrements audio ont été utilisés) et le recueil de souvenirs des témoins.

Quelques autres exemples sont brièvement convoqués de façon satellitaire. En amont, un rapide retour sur trois mises en scène de Racine permet de souligner constances et variations dans le rapport au français. En aval, un rapide survol de situations de jeu avec le rapport entre les langues donne une idée de l'importance accordée par Vitez à l'imaginaire des langues.

\section{Écrire, lire, jouer en français. Racine et Métellus}

7 Entre les années 1950 et les années 1990, en France, la façon dont a été pensé le rapport à la langue nationale et ses relations avec les autres langues a été évidemment fortement travaillée par le contexte des guerres de décolonisation, des politiques économiques de construction européenne, du développement de l'idée de francophonie et de la distinction entre aires linguistiques et aires nationales, de l'apparition du thème de l'immigration dans les médias, et des premières participations du Front national aux élections. Au théâtre, des expériences comme celles du Théâtre des 
nations (1956), du Festival mondial du théâtre de Nancy (1963), de la Maison des cultures du monde (1982), des Francophonies de Limoges (1984), ou du TILF-Théâtre international de langue française (1985), affichèrent avec détermination leur objectif: proposer au public français une ouverture internationale qui reposât sur l'invitation d'artistes étrangers.

8 Antoine Vitez soutint quelques-uns de ces projets ${ }^{9}$ et fit de la " recherche des cultures lointaines ${ }^{10}$ » l'une des cinq caractéristiques de la politique de programmation qu'il imagina lorsque, en 1979, il rédigea des propositions pour le Théâtre national de Chaillot. Devenu directeur en 1981, il affirma la nécessité de défendre sa propre langue : « Défendre sa langue, voilà un enjeu tout autant culturel que politique. Chaillot entend s'y engager sans réserve ${ }^{11}$. » Prôner une conception de la scène comme « laboratoire de la langue et des gestes de la nation ${ }^{12}$ » allait pour lui de pair, indissociablement, avec le fait de donner la parole à de nombreux artistes, acteurs, auteurs dramatiques, poètes et musiciens, dont la langue maternelle n'était pas le français.

Vitez, comme ses précurseurs, a souhaité pleinement appartenir au théâtre français et, tout à la fois, s'ouvrir à des horizons et à des collaborateurs du monde entier. Il a cultivé cette double passion de l'appartenance et de l'extension ${ }^{13}$.

9 Les acteurs de langues étrangères lui permettaient en effet d'entendre, et de faire entendre, autrement le français, de l'« étrangéiser », par la musicalité inhabituelle de leurs accents et par l'intensité de l'attention que le fait de jouer dans une langue qui n'est pas la sienne conduit à porter à la sonorité des mots. Vitez, de plus, les amenait de temps à autre à jouer passagèrement dans leur propre langue, étrangère au public ${ }^{14}$. De même, dans ses mises en scène d'œuvres traduites, ou retraduites pour l'occasion ${ }^{15}$, il collait des bribes de textes écrits dans la langue des auteurs (poèmes, chansons, extraits d'autres écrits, du même auteur ou d'un autre auteur de même langue), faisant parfois de leur diffusion et de leur écoute, à l'aide d'objets intégrés à la scénographie (radio, magnétophone) et manipulés par les acteurs, l'action centrale d'une séquence scénique.

\section{Jean Racine, « le chant parlé de la langue française »}

10 Si la matérialisation de l'altérité linguistique était continuelle dans le théâtre d'Antoine Vitez, elle prenait des formes variées, y compris avec des textes écrits en français. Le cas des mises en scène de tragédies de Racine - Andromaque (1971), Phèdre (1975), Bérénice (1980) et Britannicus (1981) - est à ce titre exemplaire d'une pratique de jeu avec l'étrangeté de la langue dont la variation était l'une des constantes ${ }^{16}$.

[...] Racine ne prescrit rien. Et c'est bien le comble de la contrainte et le comble de la liberté, une liberté qui s'apparente au vide. [...] les chemins que l'on peut prendre sont en nombre infini. [...] Naturellement cette liberté n'existe que si l'on se soumet de façon absolue à la contrainte de l'alexandrin lui-même ${ }^{17}$.

11 Rejetant la prosaïsation et affirmant le respect du vers - « Le texte de Racine n'est pas intéressant s'il n'est pas en alexandrins ${ }^{18}$ " -, Vitez expérimenta à chaque fois une forme différente de jeu avec la langue qu'il appréhendait comme inhabituelle et étrange, non seulement parce que lointaine mais aussi parce qu'inventée, « délibérément irréelle ${ }^{19}$ ».

En 1971, il appliqua à Andromaque un principe déjà mis en œuvre en $1968^{20}$ selon lequel les acteurs ne jouent pas des personnages mais jouent un texte, comme si la fable primordiale était avant tout l'histoire du rapport d'une équipe à une écriture, et la mise 
en scène un dialogue imaginaire avec un poète. Alors que la distribution traditionnelle par personnages tend à donner à la fiction une prééminence sur la poésie, ce parti pris valorise le rôle de l'écriture, et par conséquent l'importance scénique du travail de diction.

Les vers, loin d'être amoindris, prosaïsés, sont prononcés dans leur entièreté, par groupe ou séparément, de manière que le public reçoive les deux sens de la prose ancienne, transparents l'un à l'autre: d'une part le sens courant, prosaïque, des phrases qui (justement) courent le long des vers, et d'autre part le sens poétique des vers dits l'un après l'autre et toujours considérés un par un comme autant de phrases ${ }^{21}$.

13 En 1975, la mise en scène de Phèdre, fruit des recherches sur la diversité des dictions possibles entreprises dans l'atelier de rythme et de prosodie d'Ivry, étonna encore plus que ne l'avait fait Andromaque. Chanté, parlé-chanté, ou déclamé, le texte était accompagné d'épinette et d'alto: "un quasi-opéra baroque ${ }^{22}$ ", commentait Anne Ubersfeld. Henri Meschonnic, à l'aide d'un enregistrement audio conservé dans les archives personnelles d'Antoine Vitez, proposa au début des années 2000 une remarquable analyse de ce travail.

Sans du tout se travestir en opéra, Phèdre alterne, juxtapose, d'un vers ou d'une séquence de vers à un autre, des segments chantés, criés ou plus traditionnellement parlés, dits, selon des passages brusques du plus fort au plus bas, du lent au précipité.

Des mots normalement inaccentués, doublement inaccentués même, sur le plan de la langue et sur le plan métrique, se trouvent étirés démesurément, de la manière la plus antinaturaliste qui soit. Nul ne parle ainsi. Mais aussi, dans Phèdre, dans cette Phèdre, on ne parle pas : ce qu'on y entend c'est le cri interne, la bête humaine dans sa souffrance inhumaine. Plus rien de commun avec ce qu'on appelle parler ${ }^{23}$.

La distance entre la langue dans Phèdre et le français moyen des spectateurs en 1975 devenait objet d'un jeu :

La diction des douze pieds doit s'éloigner autant que possible de l'invraisemblable (oui vraiment) banalité de la diction traditionnelle. [...] nous exalterons la différence, et par exemple en jouant les petits mots, articles, conjonctions, pronoms, tout ce qui n'a pas de sens. Comme pour reconstituer un langage perdu, et en effet, il est perdu et notre reconstitution sera tout à fait imaginaire ${ }^{24}[. .$.$] .$

Ce qui est important c'est de rendre bien étranges, bien surprenantes, bien insolites ces œuvres au lieu de les rapprocher de nous, tout à fait artificiellement, par l'actualisation ${ }^{25}$.

15 Avec Bérénice (1980), Vitez, qui considérait que l'une des fonctions du théâtre est d'élever des statues aux acteurs, voulut rendre un hommage à Madeleine Marion. L'idée reposait sur une conception du théâtre comme art du portrait et sur le désir de faire une galerie de portraits croisés - des poètes, des acteurs, des personnages, les uns par les autres ${ }^{26}$. Dans une rencontre publique qui eut lieu à Francfort en 1980, il s'en expliquait ainsi, comparant ses mises en scène de Phèdre et de Bérénice :

Dans Bérénice je n'ai pas tellement repris ce travail fait dans Phèdre [...]. Au fond je voulais surtout entendre Madeleine parler. [...] j'avais envie de lui donner la possibilité de dire les vers comme elle les dit, je trouve qu'elle les dit divinement [...]. [Dans Phèdre] j'avais décalé les accents, j'avais décalé les longues et les brèves de la langue française, tandis que Madeleine au contraire utilise les longues et les brèves telles qu'elles sont enseignées par Georges Le Roy de manière absolument rigoureuse. C'est un plaisir de l'entendre parce que c'est très rare, c'est même vraiment exceptionnel. Justement c'est quelque chose qu'on n'entend plus jamais à la Comédie-Française, probablement on l'entendait autrefois ${ }^{27}$. 
Rendre hommage à une tradition en voie de disparition lui paraissait, relativement à l'histoire de la langue nationale, un acte politique.

[...] le choix de Madeleine Marion c'est le choix de quelqu'un qui fait référence à une histoire du théâtre, elle représente, pour moi, la grande actrice, la belle dame, la grande dame. Faire jouer Bérénice par Madeleine c'est faire une référence à une certaine époque du théâtre où on jouait la tragédie d'une certaine manière. Ce spectacle Bérénice inclut des époques précédentes de l'histoire du théâtre, inclut par exemple la représentation des tragédies fin $\mathrm{XVIII}^{\mathrm{e}}$, début $\mathrm{XIX}^{\mathrm{e}}$ siècle, le jeu pathétique à la Talma ${ }^{28}$.

Dans un texte écrit, portrait lui aussi, il précisait :

Madeleine Marion est dans une relation pythique avec la langue française, avec sa langue. La totalité de la construction du rôle, du personnage, des images produites par le corps, est, chez elle, déterminée par l'écoute de la langue. [...] le seul fil qui la guide, c'est, non pas la musique car la parole n'est pas de la musique, mais, je dirais, le chant parlé de la langue française ${ }^{29}$.

\section{Jean Métellus}

Antoine Vitez rencontra pour la première fois le poète haïtien Jean Métellus dans un café du Quartier latin en juin 1980 après avoir lu le manuscrit inédit de sa première pièce, Anacaona, écrite en août 1979. Touché par la qualité poétique de son écriture, par son inexpérience théâtrale, et par son rêve d'un "théâtre des commencements ${ }^{30}$ ", Vitez le programma trois fois à Chaillot, d'abord dans le cadre des «Rendez-vous de poésie » organisés par Marie Étienne en 1983, puis dans celui du " Théâtre de lecture » en 1985, et enfin en mettant en scène Anacaona dans la grande salle en 1988.

\section{«Rendez-vous de poésie »}

Lors de la soirée qui lui fut dédiée, le 2 mai 1983 (soirée enregistrée, comme la plupart des activités de Chaillot à cette période), Jean Métellus lut avec Antoine Vitez, dans le Grand Foyer, une sélection de poèmes qu'ils avaient établie ensemble ${ }^{31}$. Par-delà le plaisir et le trouble provoqués par la possibilité d'entendre les voix d'artistes disparus, le document nous permet de réaliser l'importance de l'alternance des lecteurs. Chaque passage de l'un à l'autre renouvelle en effet l'étrangeté de l'acte même de lire la poésie. À l'écoute de l'enregistrement, chaque fois que l'on entend Jean Métellus, le chant du poète paraît émaner d'une source vitale. La précision du phrasé, l'intensité du parlé s'apparentent à une danse avec les mots, enracinée dans une connaissance intime du mouvement de l'écriture. La gravité de la voix prolonge celle du verbe qui la manifeste : lecteur et poète ne font qu'un.

Son 1 - ASPBAN-001535 (29").

Jean Métellus, Brume. Voix de Jean Métellus. Fonds Antoine Vitez, BnF, Arts du spectacle. docannexe/file/2777/joinnault_son_1.mp3

Quand on entend Antoine Vitez prendre le relais, un tout autre rapport à l'écrit s'instaure, celui d'un étranger, d'un visiteur, qui sait que jamais il n'accédera à l'origine, mystérieuse, de la source, et qui, humblement, invite à écouter le chant qui en émane, délicatement en retrait, soutenu mais paradoxalement fragilisé dans l'approche du langage d'autrui par sa propre maîtrise technique de l'acte de dire, par son savoir d'acteur. Dans le rapport de celui qui dit à celui qui amicalement le cite, assumant de 
mettre en jeu sa propre capacité d'écoute du souffle d'autrui et sa singularité de lecteur, l'auditeur mesure la difficulté de l'exercice, ce devoir et cette impossibilité, non seulement de traduire et de représenter, dont Vitez se faisait le chantre, mais aussi d'écouter et de donner à écouter. Comme sujet et comme thématique, la sensorialité auditive était d'ailleurs présente dans plusieurs des "Rendez-vous de poésie » qui furent programmés à Chaillot, dessinant en creux un éloge de l'attention.

L'ouie dans le corps s'est incrustée comme le plus pudique des sens, ne laissant au monde comme tout repère qu'un cartilage dressé comme une grande question des deux côtés de la tête

[...]

Dans le tendre âge chante l'ouie, je suis caresse et graine. L'ouïe chante et vit comme une sauterelle dans l'âge de tout repos. L'ouïe toujours occupée, toujours aux aguets, l'ouïe aux longues antennes ${ }^{32}$.

Son 2 - ASPBAN-001535 (35")

Jean Metellus, Au pipirite chantant. Voix d'Antoine Vitez. Fonds Antoine Vitez, BnF, Arts du spectacle.

docannexe/file/2785/joinnault_son_2.mp3

Son 3 - ASPBAN-001535 (29")

Jean Metellus, Au pipirite chantant. Voix d'Antoine Vitez. Fonds Antoine Vitez, BnF, Arts du spectacle.

docannexe/file/2792/joinnault_son_3.mp3

\section{"Théâtre des commencements »}

21 Long poème versifié, dialogué, structuré en quatre actes et vingt-cinq scènes, Anacaona raconte l'extermination des Indiens d'Haïti, de l'arrivée de Colomb en 1492 à l'assassinat de la reine-poète du Xaragua, Anacaona, par le gouverneur Ovando en 1503. La langue et la parole jouent un rôle central dans le récit. Tirant leçon de la gloire posthume du Christ, les missionnaires catholiques prétendent en effet que l'accomplissement de leur projet nécessite une transformation radicale de la manière de penser, d'agir, et de percevoir des Indiens; transformation qui passerait par une évangélisation musclée et par un apprentissage forcé de l'espagnol : «[...] là où il faut atteindre ces sauvages / Qui s'en vont nus comme jadis au paradis / C'est dans leur âme, dans leur être profond ${ }^{33}$ ". Considérant que "dans ces pays encore sauvages la parole est une arme redoutable / Elle remplace l'épée et les canons ${ }^{34}$ ", les colons voient la reine-poète comme une ennemie suprême, "le cerveau du pays ${ }^{35}$ ", celle qui sait parler à son peuple, et même, charmer certains Espagnols. Aussi est-ce justement à la parole qu'ils recourent, profitant de la surdité d'Anacaona et de Caonabo, le roi, aux prophéties divines, pour les abuser et les détruire.

Le texte est réparti entre onze personnages, sept Indiens ${ }^{36}$ et quatre Espagnols ${ }^{37}$. Pendant les deux premiers actes, les dialogues, qui se déroulent exclusivement entre Indiens ou entre Espagnols, ne mettent en scène aucun conflit interpersonnel mais décrivent les situations historiques et dressent des portraits. À partir du troisième acte, lorsque, dans la deuxième scène, Anacaona et Yaquimex tentent de se convaincre mutuellement de la stratégie à adopter après le massacre de la Vega Real, la parole, jusqu'alors narrative et lyrique, devient action et manifestation du trouble de la Reine et de la résistance de Yaquimex.

Anacaona. - Écoutez bien

Quand la force déploie l'éventail de sa voix et de son charme, exhale ses vapeurs

déréglées sur une terre et des hommes exténués 
J'arbore les vertus de la sagesse et prodigue l'enseignement de la patience

Yaquimex. - (En écho)

Anacaona. - Que le métier de diriger est dérisoire

Reine sans pouvoirs ou roi de batos,

Tout cela se ressemble

Yaquimex. - (En écho)

Anacaona. - Ce n'est pas la prière, c'est quelque chose qui m'est passé dans l'esprit en pensant à la réunion de demain où il faut vingt avis pour prendre une seule décision

Yaquimex. - (En écho)

Anacaona. - Vous ne semblez plus me suivre

Tâchez de répéter seulement mes paroles sensées

Et de vous taire dès qu'il s'agit de réflexions intimes

Yaquimex. - (En écho)

Anacaona. - Vous ne m'écoutez plus. Il faut que je contrôle ma langue.

Le poète déborde la reine.

Yaquimex. - (En écho et il ajoute, largement, très largement)

Anacaona. -Revenez demain. Car notre entretien vous a fatigué

Vous répétez mais vous suivez une autre pensée

Yaquimex. - (En écho et il ajoute, très intime, très intime $\left.{ }^{38}\right)$

Son 4 - ASPBAN-001342 (1'07")

Jean Metellus, Anacaona. Laurence Roy (Anacaona), Frédéric Merlo (Yaquimex). Fonds Antoine Vitez, BnF, Arts du spectacle.

docannexe/file/2799/joinnault_son_4.mp3

Les dialogues entre Indiens et Espagnols ont lieu dans le dernier tiers de la pièce. Alors que la brutalité des destructions physiques est rapportée par des récits, le piège de la parole par lequel va succomber la reine-poète est directement montré. Les Indiens massacrés, la reine pendue, Yaquimex s'enfuit dans les montagnes en chantant « Aya bombé, Aya bombé39 $"$.

\section{" Théâtre de lecture »}

24 La tragédie de Métellus pose à la scène de nombreux problèmes concrets : sa longueur, son lyrisme, la faible activité dramatique de ses personnages qui sont la plupart du temps transformés en messagers, la difficulté de représenter un peuple éradiqué il y a presque quatre siècles. Le 25 février 1985, dans le Grand Foyer, Antoine Vitez en lut de larges extraits qu'il avait sélectionnés avec Anne-Marie Métellus. L'archive sonore de la soirée débute ainsi :

Avant de commencer à lire je fais un petit rangement. Voilà, je suis content que vous ne soyez pas tout à fait dans le noir car je préfère voir les gens à qui je m'adresse par la lecture. On a joint au programme qu'on vous a donné ici une feuille. Cette feuille vous permet éventuellement de suivre un certain nombre de noms et de lieux géographiques. 
(on entend alors des bruits de feuilles)

J'ai l'impression (rires) de faire un cours. Mais vous n'êtes pas obligés de l'apprendre (rires). Éventuellement puisque vous avez un peu de lumière ça vous évitera de vous perdre. De toute façon il n'y a à mon avis pas lieu de se perdre parce que le déroulement de la tragédie doit avoir sa logique en lui-même.

Alors voilà j'ai mis ici un certain nombre d'objets, et ces objets ce sont, si vous voulez, des personnages, c'est-à-dire n'ayant pas la possibilité de jouer avec des acteurs en chair et en os, je vais utiliser ces objets pour représenter de temps en temps, pas tout le temps, quand c'est nécessaire, pour représenter l'action.

Alors. Acte premier, scène première, Anacaona. En scène il y a un seul personnage (on entend des bruits d'objets métalliques que l'on fait bouger et le bruit sourd de l'un d'entre eux que l'on pose ostentatoirement sur la table, probablement pour représenter Altabeira) qui est la confidente d'Anacaona.

Alors ceci est le bord de la scène. C'est Altabeira, la confidente d'Anacaona. Voici ce que dit Altabeira. On peut imaginer qu'elle parle d'abord toute seule et qu'Anacaona n'est pas là. Ou bien on peut imaginer une autre mise en scène et qu'Anacaona est présente un peu derrière et au centre de la scène pendant que la confidente parlera le visage tourné vers le public. Mais je préfère penser qu'elle n'est pas là, elle va venir.

Altabeira $^{40}$.

Temps de contact attentif, accompagnement détendu des spectateurs, exposition des règles du jeu, ce prélude dans lequel celui qui va lire un long poème commence par prendre le temps d'improviser un discours qui lui permet d'installer les conditions de l'écoute illustre bien la délicatesse de Vitez qui cherchait à se tenir à distance, à ne pas happer le spectateur dans la contemplation de sa propre performance de lecteur (voix, corps, souffle), mais à susciter son imagination, prolongeant l'idée d'une œuvre rêvée pour le théâtre, faisant du bord de la table un bord de scène, d'un objet un personnage $e^{41}$, conviant les spectateurs à prendre appui sur ces quelques éléments pour se figurer eux aussi une scène imaginaire. Au cours des un peu plus de deux heures de ce théâtre de lecture, il ne cessa de suspendre l'enchaînement des dialogues, indiquant le découpage en actes et en scènes, annonçant le nom d'un personnage ou même le registre de ses propos (« et on va entendre la première prophétie d'Altabeira »), lisant une didascalie comme s'il décrivait concrètement une action scénique, résumant une scène au lieu de la lire ( scène 3 , je la résume. Anacaona, Caonabo et Altabeira. Au cours de cette scène, Anacaona annonce la ruine de la Nativité - campement construit par les Espagnols, par la faute de Guakanagarik. Caonabo lui dit "Il faut maintenant convaincre votre frère Bohéchio / Que l'homme ne voyage jamais pour rien / Et qu'on ne vient pas du bout du monde / Pour apporter le message d'un dieu invisible". Et Caonabo décrète le $\left.\operatorname{combat}^{42} »\right)$. De la formulation d'hypothèses scéniques à la description d'une situation, de la lecture intégrale d'une scène (pour les deux tiers du texte environ) au bref résumé d'une autre, en passant par des traitements mixtes, il empruntait tour à tour à l'art du conteur, du marionnettiste, de l'acteur ou du metteur en scène. À l'écoute de l'enregistrement, il semble que, dans la tension entre impossibilité et désir de lire, ces continuelles variations, loin de détourner de l'écoute du texte de Métellus, ne cessaient de renouveler et de stimuler l'attention. 


\section{Mise en scène} Soulier de satin dans la grande salle de Chaillot, eut lieu, « comme un écho discordant ${ }^{43}$ ", la première d'Anacaona : «Ici on entend la réponse de l'homme des Caraïbes au discours conquérant de Colomb et de Cortès. "Élargir le monde", dit Don Rodrigue. Oui, certes, mais par le massacre et la soumission. "Qui composera pour nous ballades et ballets ?", s'écrie l'Indien, tourné vers l'avenir, c'est-à-dire vers nous ${ }^{44}$ ». Le spectacle fut présenté dans la grande salle en alternance avec Le Misanthrope. " Nulle relation entre la tragédie haïtienne de Métellus et le Misanthrope, aucun lien de sens, et rien dans cet accouplement-là n'est à démontrer. [...] Point de honte à utiliser Molière pour aider Métellus, il le lui doit ${ }^{45}$ ».

L'archive sonore consultée est celle d'une représentation intégrale ${ }^{46}$. Elle commence par un solo de clavecin préparé qui surprend immédiatement. En effet, bien qu'il existe des traces de musique baroque écrite au XvII ${ }^{\mathrm{e}}$ siècle pour être jouée par des orchestres d'Indiens dans des écoles de jésuites, le clavecin semble n'entretenir aucun lien évident ni avec l'époque de l'extermination racontée, ni avec celle de l'écriture de la pièce sous la dictature de Jean-Claude Duvalier (1979), ni avec celle de sa mise en scène en pleine période d'instabilité politique en $\mathrm{Haiit}^{47}$, ni non plus avec les références musicales les plus communément associées aux cultures espagnole ou antillaise par un public français de la fin du $x^{e}$ siècle. Ce solo interprété par David Recoing ${ }^{48}$ nous transporte d'emblée dans le contexte des explorations menées par Georges Aperghis dans les décennies 1970 et $1980^{49}$, celui d'un jeu contemporain avec un instrument ancien et avec un répertoire partiellement déformé, comme dans un rêve ou dans un souvenir. Des structures rythmiques, des accords, des mélodies étirées et désintégrées évoquent, par touches, le répertoire baroque, à certains moments son raffinement et sa rigueur, à d'autres ses ornements et sa préciosité. L'étonnement initial est constamment réactivé par les variations inattendues de la partition, composée de fragments déstructurés et dissonants et de séquences rigoureusement mesurées et consonantes. Le rapport au passé et à l'ailleurs joue du vague, des approximations de la mémoire et des imaginaires. Entre les premiers vers de la pièce prononcés par Pascaline Pointillard «Le sang, les larmes submergent le Marien / Ce pays vêtu de défroques de monstres marins / Recouvert par les débris d'un canot gigantesque / Envahi par des flots de chrétiens enivrés / N'a plus son roi Guakanagarik» - et l'ouverture au clavecin, la relation est avant tout de l'ordre du rapport aux langages. C'est en effet à l'écriture même de Jean Métellus, dans laquelle Antoine Vitez percevait un imaginaire de la majesté de la langue française proche de celui qui caractérise les grandes œuvres du répertoire francophone européen, celle de Racine en particulier, qu'il eut l'idée d'associer le clavecin, symbole du prestige et de la musique européenne des $\mathrm{XVII}^{\mathrm{e}}$ et $\mathrm{XVIII}^{\mathrm{e}}$ siècles. Cette association situait d'emblée la mise en scène dans un registre de sublimation du chant des mots.

Anacaona est une tragédie véritable, de forme classique, écrite en une langue très pure, qui est la marque de la poésie antillaise, héritière - on ne le dit jamais - de Racine plus que de Shakespeare: le poète se soucie peu de distinguer les personnages selon leur extraction, leur condition ou leur caractère, par les mots dont ils usent; ici le paysan parle comme son seigneur; tout est dans l'Esprit, l'idée; la beauté uniforme de la langue appartient au poète seul, qui la distribue également à chacune de ses créatures. Cette particularité de la poésie antillaise (de 
Césaire à Jacques Stéphen Alexis, ou Métellus, ou Lemoine) est le témoignage d'une revendication classique, par quoi elle affirme en face de nous sa dignité ${ }^{50}$.

fait que deux des acteurs, Murray Grönwall avec son alto ${ }^{51}$ et Pascaline Pointillard par le chant, glissaient parfois légèrement dans le registre musical, créaient des effets de circulation et d'unité entre parole et musique. Ainsi, dans la transition entre les deux premiers actes, entre l'exposé du point de vue des Indiens (fin de l'acte I) après la victoire de Caonabo et l'exposé de celui des Espagnols qui préparent déjà l'agression suivante (début de l'acte II), Grönwall prolongeait les accords du clavecin par un jeu pizzicato. Bien que consonant, le duo clavecin-alto semblait étrange et chaotique, comme s'il disait la cohabitation entre deux univers difficilement conciliables. Un peu plus tard, avant que Caonabo n'ait été capturé, l'acteur interprétait un lamento sans doute inspiré d'une mélodie populaire scandinave. La voix de l'alto, tel un chœur tragique, se faisait annonciatrice d'un événement funeste. Pascaline Pointillard, quant à elle, exécutait à plusieurs reprises des vocalises filées. Dès le début du spectacle, au bout d'une trentaine de secondes de clavecin, la voix entrait ainsi en scène, d'abord de façon non verbale. Dans la succession instrument/chanté/parlé, les vocalises créaient un lien fluide et charnel entre les notes et les mots ${ }^{52}$.

30 La diction jouait elle aussi de l'irrégularité, une irrégularité qui servait à matérialiser des différences entre personnages et à mettre en relief leurs singularités. Pascaline Pointillard, l'interprète d'Altabeira, étrangéisait la langue, suspendait brusquement le flux puis précipitait le débit, égrenait des mots isolés ou les groupait en grappes, multipliait d'imprévisibles changements de volume, glissait dans l'inarticulé, comme si la messagère des dieux se situait dans un entre-deux, entre langage céleste et langage terrestre. Grégoire Ingold traduisait l'assurance flamboyante et solaire du roi-guerrier Caonabo par d'amples modulations et par une diction régulière, vigoureuse et souple, qui donnait à entendre la mesure, les longues et les brèves, les rimes internes, les consonances. Laurence Roy donnait chair à la force incantatoire de la reine Anacaona, à sa conscience de poète. Dans un corps à corps littéralement extra-ordinaire avec la matière-langue, elle rythmait, sculptait, exacerbait les reliefs, scandait, suraccentuait, séparait, étirait, frappait, sifflait, chantait, multipliait les effets de rimes, comme si, d'elle, la poésie jaillissait. Frédéric Merlo, au contraire, par une diction sans heurts, claire et économe, faisait entendre la détermination lucide de Yaquimex, sa résistance critique. 
Son 5 - ASPBAN-001341 (52")

Jean Metellus, Anacaona. David Recoing (au clavecin), Pascaline Pointillard (Altabeira). Fonds

Antoine Vitez, BnF, Arts du spectacle.

docannexe/file/2807/joinnault_son_5.mp3

Son 6 - ASPBAN-001341 (1' 20")

Jean Metellus, Anacaona. Grégoire Ingold (Caonabo). Fonds Antoine Vitez, BnF, Arts du spectacle. docannexe/file/2813/joinnault_son_6.mp3

Son 7 - ASPBAN-001342 (46")

Jean Metellus, Anacaona. Laurence Roy (Anacaona). Fonds Antoine Vitez, BnF, Arts du spectacle. docannexe/file/2821/joinnault_son_7.mp3

31 Ni l'écriture, ni le jeu en français, ni les apports du clavecin n'occultaient les origines du poète et de l'histoire racontée. Les acteurs faisaient en effet chanter les sonorités caraïbes dont le texte est amplement émaillé ${ }^{53}$. La déclamation soulignait leurs présences, leur éclat, ainsi quand la reine appelle les caciques à venger Caonabo, Laurence Roy semble parler, en français, une langue étrangère.

Dites à Yaquimex

De rejoindre Manicatex, Guarionex et Mayobanex

Avec toutes les forces du Xaragua

Pour attaquer Isabella ${ }^{54}$.

Son 8 - ASPBAN-001341 (10")

Jean Metellus, Anacaona. Laurence Roy (Anacaona), David Recoing (au clavecin). Fonds Antoine Vitez, BnF, Arts du spectacle. docannexe/file/2829/joinnault_son_8.mp3

Par contre, alors que Métellus avait réservé aux Indiens le "Aya bombé, Aya bombé ${ }^{5}$ ", chant de révolte, symbole du refus de la soumission, répété une vingtaine de fois tout au long des quatre actes et par lequel la pièce se termine ${ }^{56}$, Vitez choisit de le faire circuler, l'insérant, par collage, dans la partition de Murray Grönwall et dans celle de David Recoing. Ainsi au tout début du deuxième acte, dans la première scène où le Frère Buyls expose au gouverneur Hodeja les mesures d'évangélisation qu'il souhaite imposer (faisant ainsi du clergé un partenaire crucial de l'entreprise coloniale), Pierre Romans, qui jouait Hodeja, se mettait à "glousser » tandis que Murray Grönwall énonçait les propositions humiliantes et haineuses du missionnaire. Entre les unités de souffle et pendant les suspensions de la parole en cours de vers, son rire se substituait au silence, puis passait au second plan quand le texte reprenait. Finalement Grönwall se mettait à rire lui aussi et son rire se muait en " Aya bombé », puis en « Aya », comme si ce simple mot symbolisait à lui seul la langue et la culture de l'autre, sa différence.

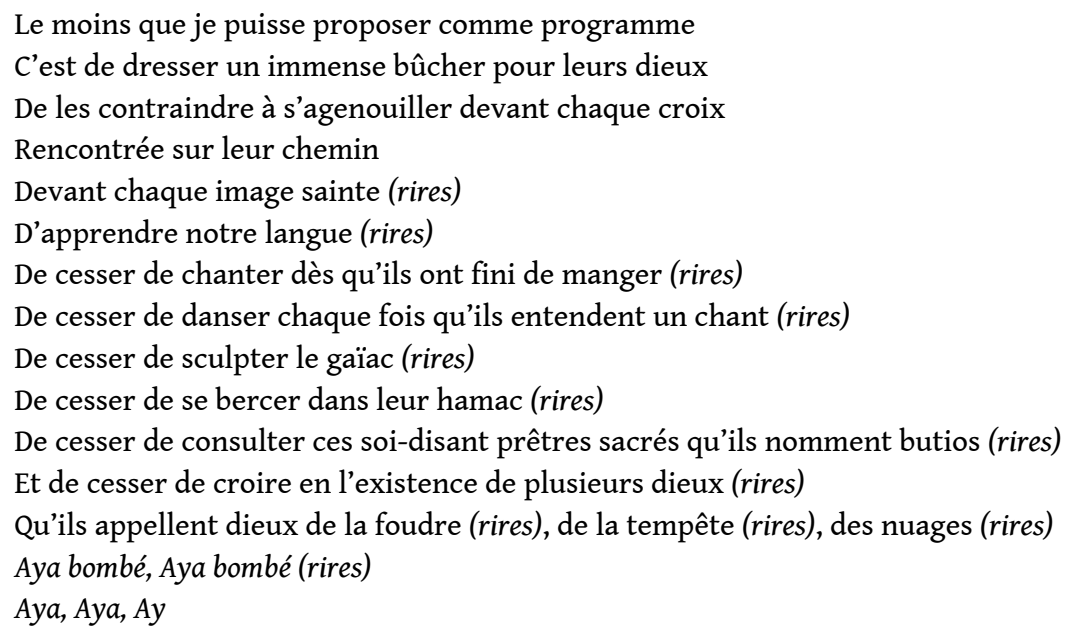


Cette terre, Hodeja, est une terre de feu

Si les mesures que je propose ne sont pas acceptées

Nous serons obligés de partir

Après avoir pourtant conquis

Refondre une mentalité demande de la méthode ${ }^{57}$.

autre circulation se faisait simplement par dissociation du texte et de la mélodie qui lui avait été associée précédemment. Quelques notes pianotées suffisaient alors à rendre sa présence diffuse en arrière-plan, telle une ritournelle des origines. $\mathrm{Ce}$ détachement du texte de ses énonciateurs historiques, et même, des corps vivants des acteurs, accentuait le passage de l'histoire à la mémoire, faisant du «Aya bombé » un trésor universel, un chant mythique, une survivance de la destruction d'un peuple et d'une culture dont il appartiendrait à tous, par delà les différences et les époques, de raconter l'histoire.

Son 9 - ASPBAN-001341 (1'03")

Jean Metellus, Anacaona. Frédéric Merlo (Yaquimex). Fonds Antoine Vitez, BnF, Arts du spectacle. docannexe/file/2835/joinnault_son_9.mp3

\section{Transfert de mémoire}

Dans un débat de 1988 consacré à cette première mise en scène d'une première pièce, Antoine Vitez, réagissant à l'enchantement d'une spectatrice émerveillée qui lui demandait de préciser en « quelle mesure le choix de la pièce avait été déterminé par la beauté du langage de Métellus » qu'elle avait découvert grâce au spectacle, en quoi ce choix avait été "difficile ou spontanés8 ", répondit d'abord en disant que ce qui le retenait toujours quand il lisait était la langue, en particulier la langue française, sa langue, puis il raconta comme il pouvait être bouleversé, frappé par la qualité poétique des écritures, des dialogues brefs de Vinaver au long poème dramatique de Métellus.

Son 10 - ASPBAN-001345 (1' 58")

Une spectatrice à Antoine Vitez, débat après le spectacle Anacaona. Fonds Antoine Vitez, BnF, Arts du spectacle.

docannexe/file/2840/joinnault_son_10.mp3

Vint ensuite un long échange autour de la représentation des Indiens : le lyrisme du texte, la beauté de la scénographie, le jeu des acteurs n'éloignaient-ils pas les faits historiques, ne les déréalisaient-ils pas ? s'interrogeaient certains spectateurs.

Ce qui est bizarre dans notre entreprise, ce qui m'a intéressé, c'est que les acteurs qui jouent sont des Français d'aujourd'hui et que la pièce a été écrite par un noir parlant d'autres gens, ni noirs, ni blancs, d'avant, ce que je trouve intéressant c'est ce double transfert, le théâtre sert à représenter des choses que rien d'autre vraiment ne peut représenter. [...] c'est la jouissance historique, la jouissance de la résurrection aussi, on fait resurgir des personnages qui ont existé il y a tant de temps, [...] on peut toujours le faire dans d'autres langues, avec une autre peau, on n'a pas la même couleur, on n'a pas la même voix, mais on refait quand même les mêmes gestes... c'est cette idée du transfert de mémoire ${ }^{59}$.

Et de nouveau la question de la langue, du choix de la langue, surgissait. Pourquoi ne pas avoir écrit et ne pas jouer en espagnol, langue de ceux qui colonisèrent Haïti, langue des archives historiques consultées pour écrire la pièce ? Pourquoi ne pas avoir écrit en créole, langue maternelle du poète et langue des Haïtiens de 1988 qui leur permettrait, plus que le français, d'accéder au théâtre? Vitez affirmait simplement 
qu'il mettait en scène Anacaona parce qu'il trouvait le texte remarquablement beau et ambitieux, et souhaitait, le faisant avec des acteurs français sur une scène française, le faire connaître au public français. Métellus disait qu'il avait écrit dans sa langue seconde, langue officielle en Haïti depuis 1804, par fidélité à ses racines et à son peuple, parce que c'était, pensait-il, au moment où il l'avait fait, la meilleure façon de faire connaître son pays. Dans un article intitulé «Une parole haïtienne dans la langue française ${ }^{60} »$, il précisait :

L'usage d'une langue autre que la langue maternelle n'indique pas forcément déchirement ou tensions. Les problèmes sont d'ordre social, politique et peut-être, de nos jours encore, racial. L'aliénation doit être recherchée parmi ces derniers paramètres et non dans la pratique d'une langue ou d'une autre ${ }^{61}$.

Refusant d'associer l'aliénation au choix de la langue, il défendait l'idée qu'écrire librement dans une langue qui n'est pas la sienne oblige à déployer des efforts fructueux qui sont source d'enrichissement et de fortification.

\section{Entre les langues}

Un des cas les plus frappants, et les plus comiques, du jeu avec le rapport entre les langues est sans doute celui de La Rencontre entre Pompidou et Mao (1979) où, partant d'un texte en français publié dans Le Nouvel Observateur et donné par la revue comme la transcription d'une rencontre historique entre les deux chefs d'état, Antoine Vitez mit en scène deux personnages de traducteurs muets qui intervenaient constamment pour traduire ce que les présidents se disaient. Par des mimiques corporelles grotesques, ils exhibaient la difficulté de passer d'une langue à l'autre, détruisant avec malice l'illusion d'adresse directe produite par le journal.

Ce désir de donner à percevoir la traduction, ses inévitables approximations et ses multiples variations potentielles, s'exprimait également de façon privilégiée dans les «Rendez-vous de poésie » qu'organisait Marie Étienne à Chaillot. L'archive sonore de l'enregistrement d'une soirée consacrée au poète Guennadi Aïgui (1934-2006) en juin 1982 illustre bien cette idée. Un chant polyphonique en tchouvache ouvrait la soirée, donnant à écouter la langue maternelle du poète. Puis des poèmes, écrits en russe et traduits en français, étaient lus alternativement par Léon Robel, le traducteur, et par Antoine Vitez. Au cours de la séance, Vitez raconta aux auditeurs qu'il aurait aimé traduire lui aussi des poèmes d'Aïgui, de sorte à ce que la soirée prît la forme d'un exercice de joute littéraire entre traducteurs et donnât à entendre, pour un même poème, plusieurs alternatives de traduction. Il n'en avait pas eu le temps, précisait-il, mais il lut néanmoins un poème deux fois de suite, la première en russe, la seconde en français.

[Lien au dossier pédagogique multimédia en ligne « Entendre le théâtre », à venir début juillet 2019]

40 L'exemple est assez simple, mais l'engagement voluptueux dans la lecture du poème en russe, le corps à corps exalté avec la langue de l'autre, la sensualité de la déclamation, situent une posture qui est à la fois d'humilité et de désir fiévreux dans l'approche des langues et de la diction, dimensions que l'on oublie parfois dans l'abord, d'emblée plus polémique, du théâtre. Pourtant leur constance, ainsi que celle de l'affirmation du devoir et de l'impossibilité de traduire, à l'écrit comme à l'oral, donc de jouer en 
français, étaient des fondations inébranlables sur lesquelles reposaient les mises en jeu, parfois jugées incompréhensibles, voire déplacées, du rapport des langues.

Dans ses mises en scène abordant l'histoire nationale, Vitez s'engagea dans trois voies complémentaires pour mettre en scène les contacts linguistiques issus du fait colonial : faire vibrer avec ferveur la force poétique du français langue seconde des auteurs et des acteurs d'Afrique, des Antilles, de la Méditerranée ; traiter la fatuité des dominants sur un mode grotesque, exhibant leur haine et leur surdité; donner à entre-entendre délicatement, musicalement, comme un joyau rare, la langue maternelle de l'étranger méprisé. La mise en scène d'Anacaona illustre la première de ces trois voies. Pour les deux autres, je donnerai deux exemples issus de la première saison à Chaillot (1981-1982), un exemple du registre grotesque avec Tombeau pour 500000 soldats et un exemple du registre délicat avec Entretien avec Monsieur Saïd Hammadi, ouvrier algérien. Dans les deux cas, le texte original est écrit en français, par Pierre Guyotat pour le premier, par Tahar Ben Jelloun pour le second et c'est par une invention de mise en scène que la langue de l'autre, enregistrée, était donnée à entre-entendre. Entreentendre comme on dit entre-apercevoir, brièvement, mais entre-entendre aussi, d'une part, parce que l'écoute au théâtre est partagée et, d'autre part, parce que Vitez la mettait en abyme, le public étant, simultanément, mis en position d'entendre la langue maternelle de l'autre et d'être spectateur de l'écoute, jouée par les acteurs, des voix étrangères.

Dans Tombeau, des "voix arabes» enregistrées et progressivement amplifiées provoquaient des rires troupiers des acteurs sur le plateau, rires que venait recouvrir une musique chargée de dire la douleur des suppliciés. Le tuilage des plans sonores et les coupures abruptes créaient des effets oppressants de placage et d'étouffement. Dans Hammadi, une petite cassette présentée comme le support d'une correspondance orale entre Hammadi et sa fille Houria servait à faire entendre la voix de la jeune fille. Des jeux muets de manipulation du matériel et un faux démarrage préparaient la diffusion du message. S'il ne connaissait pas la langue enregistrée, le spectateur, devenu un instant étranger, était conduit à se concentrer sur le jeu des acteurs incarnant des personnages absorbés par l'écoute intensive de paroles dont le sens lui échappait. Dans ces deux exemples, les voix enregistrées pour composer les bandes-son étaient celles de locuteurs parlant dans leur langue maternelle mais elles n'étaient pas, pour autant, imitation vraisemblable et fictionnellement cohérente de voix de personnages. Le message d'Houria par exemple était en arabe dialectal marocain, la langue de Ben Jelloun, alors que le personnage de l'entretien est kabyle, et les propos enregistrés, pour ceux, rarissimes, qui les comprenaient, ne pouvaient guère passer pour des propos tenus à son père par une enfant. Comme dans La Rencontre entre Pompidou et Mao et ses figures de traducteurs, la lutte contre l'illusion d'un accès direct, facile, voire assimilateur, à la langue de l'autre se faisait en mettant en jeu la distance qui nous en sépare. C'est un imaginaire des langues, volontairement approximatif, qui était activé, dans une démarche semblable à celle d'Aperghis avec le clavecin d'Anacaona.

[Liens au dossier pédagogique multimédia en ligne "Entendre le théâtre ", à venir début juillet 2019]

La mise en scène du rapport à la diversité linguistique passait également par le fait que des acteurs parlaient ou chantaient dans des langues qui n'étaient pas les leurs. L'enregistrement d'une table ronde organisée par le Goethe Institut de Paris montre que cette liberté prise avec la langue d'autrui interpellait certains spectateurs. À propos 
de la prononciation de Boguslawa Schubert qui, dans la deuxième mise en scène de Faust (1981), se mettait à jouer en allemand le texte de Marguerite après que celle-ci avait été traitée de catin par son frère Valentin, un spectateur observait : « S'il y avait des Allemands dans la salle parmi les spectateurs, cet allemand-là, parlé de cette façon, ne pouvait que leur hérisser le poil, quant aux Français ils ne le comprenaient pas, alors pourquoi ? Est-ce que ça a un sens ${ }^{62}$ ?» Vitez ne voyait pas pourquoi, alors que l'actrice polonaise jouait en français, il aurait été insensé qu'elle jouât en allemand. Que les acteurs délaissassent par moment la traduction pour prononcer le texte originel, ou qu'ils se missent à chanter en allemand, mais aussi en suédois, en néerlandais, ou en anglais, participait d'un jeu avec un imaginaire des « cultures germaniques » et avec un texte que Vitez abordait comme l'une des grandes œuvres du répertoire mondial qu'un théâtre populaire pouvait, grâce aux traducteurs et aux acteurs, porter au public, dans une démarche consciemment approximative et volontairement ludique.

\section{« Il faut apprendre à écouter la langue »}

Mise en jeu de l'écoute, mise en jeu de la traduction, mise en jeu des liens entre l'articulé et le vocalique, ces quelques exemples montrent très concrètement qu'un véritable imaginaire des langues et des voix était à l'œuvre dans le théâtre de Vitez. La variété de ces matérialisations scéniques servait une poétique de l'attention qui nous rappelle que l'affirmation de l'amour de sa propre langue, l'investigation de ses possibles rythmiques et prosodiques, n'est en rien incompatible, bien au contraire, avec une célébration, sans hiérarchisation, de la diversité des langues et du désir d'écoute.

Il faut apprendre à écouter la langue, sa propre langue, la Langue en général, toutes les langues, tous les accents, les parlers, tous dignes d'attention, aucun moins pur ou moins beau que les autres, c'est le miracle de la multiplication des pains. La parole est la seule chose que l'on puisse partager infiniment, il en restera toujours ${ }^{63}$.

À deux reprises Daniel Soulier a mis en scène un montage, réalisé avec Jeanne Vitez, d'extraits des Conversations d'Émile Copfermann. La deuxième fois, en 2003, à la Comédie-Française, c'est le seul acteur noir de la troupe, de langue maternelle bambara, Bakary Sangaré, qu'il a choisi pour jouer Vitez :

Il est tout ce qu'aimait Antoine Vitez: l'étranger! L'autre! Quelqu'un à comprendre, à écouter. Antoine aimait par-dessus tout les voix étrangères, le français parlé par une voix étrangère. Il lisait dans l'écoute attentive des accents étrangers le destin des hommes, l'histoire de notre pays, et par extension, l'histoire de l'humanité ${ }^{64}$.

[Lien au dossier pédagogique multimédia en ligne « Entendre le théâtre », à venir début juillet 2019]

\section{NOTES}

1. Antoine Vitez, « Mon ami Aïgui », Le Monde des livres, 18 mai 1984, repris dans Antoine Vitez, Le Théâtre des idées, anthologie réalisée par Georges Banu et Danièle Sallenave, Paris, Gallimard, 
[1991] 2015, p. 47. Pour les citations de textes repris dans l'anthologie, nous indiquerons désormais TDI, suivi du numéro de page, par exemple: TDI, 47. Nous utiliserons systématiquement la réédition de 2015 qui comporte une préface ajoutée.

2. Antoine Vitez, «Incarner les fantômes », Le Journal de Chaillot, n 10, février 1983, p. 10.

3. Émile Copfermann, Conversations avec Antoine Vitez (de Chaillot à Chaillot), Paris, P.O.L, [1981] 1999 , p. 54.

4. Antoine Vitez, « Le devoir de traduire », entretien avec Georges Banu, Alain Girault et François Rey, retranscrit par Michèle Raoul-Davis, Théâtre/Public, n 44, mars 1982, TDI, 296.

5. Georges Banu et Danièle Sallenave, « Note liminaire », TDI, 10.

6. Georges Banu et Danièle Sallenave, « De la traduction généralisée », TDI, 585.

7. Voir Antoine Vitez, «Le devoir de traduire ", loc. cit., TDI, 287-298; Georges Banu et Danièle Sallenave, «De la traduction généralisée », TDI, 585-590. Voir aussi les témoignages et analyses de Georges Banu, Léon Robel, Chrysa Prokopaki, Henri Meschonnic et Éloi Recoing in Jean-Michel Déprats (dir.), Antoine Vitez, le devoir de traduire, Arles, Actes Sud, « Apprendre », [1996] 2017.

8. Il a ainsi fallu des années de travail et d'insistance historiographique pour que le succès de Catherine - spectacle souvent présenté à tort comme une œuvre fondatrice (selon une tendance qui consiste à confondre œuvre fondatrice et œuvre exemplaire) - ne serve plus à faire oublier tout le travail antérieur d'Antoine Vitez avec les textes non dramatiques, et à occulter ses enjeux poétiques et politiques.

9. Gabriel Garran m'avait confié lors d'un entretien que, lorsqu'il avait voulu créer le TILF, il avait écrit à tous les directeurs de théâtre en Île-de-France et n'avait reçu que deux réponses, parmi lesquelles celle de Vitez, qui l'avait soutenu en accueillant dans la salle Gémier, en octobre 1985, ce qui avait constitué la première réalisation du TILF : sa mise en scène de Je soussigné cardiaque de Sony Labou Tansi.

10. Antoine Vitez, «Positions et propositions pour le Théâtre national de Chaillot », 21 août 1979, Écrits sur le théâtre, t. 5, P.O.L, 1998, p. 144.

11. Antoine Vitez, "Le théâtre, lieu où le peuple vient écouter sa langue », Journal de Chaillot, $\mathrm{n}^{\circ} 6$, juin 1982, p. 8 .

12. Ibid.

13. Georges Banu, « Le legs de l'écrit » (préface), TDI, I.

14. Ces parenthèses pouvaient aller de brefs moments où les acteurs, exprimant l'état émotionnel d'un personnage, se mettaient à chantonner dans leur langue natale, à de véritables scènes comme dans la remarquable leçon de dogon conduite par Akonio Dolo dans Vendredi ou la vie sauvage (1973). Voir sur ce point Brigitte Joinnault, « De l'intérêt de certaines leçons de dogon et des travaux de l'anthropologie théâtrale dans la pédagogie des pratiques de l'oral en français ", in Christophe Alix, Dominique Lagorgette, Ève-Marie Rollinat-Levasseur (dir.), Didactique du français langue étrangère par la pratique théâtrale, Chambéry, université de Savoie, 2013, pp. 57-66.

15. Par Antoine Vitez lui-même ou par d'autres artistes (Raymond Lepoutre, Florence Delay, Éloi Recoing, André Markovitch notamment).

16. Pour une analyse ample et approfondie des mises en scène des tragédies de Racine, voir Anne-Françoise Benhamou, «Les mises en scène de Racine de Copeau (1937) à Vitez (1981)", thèse sous la direction de Bernard Dort, université Paris 3, 1983.

17. Antoine Vitez, entretien avec Henri Meschonnic, «À l'intérieur du parlé, du geste, du mouvement ", Langue française, $n^{\circ} 56,1982$, p. 31.

18. Antoine Vitez, in Émile Copfermann, Conversations avec Antoine Vitez, op. cit., p. 152.

19. Ibid., p. 105.

20. Dans la mise en scène de La Grande Enquête de François-Félix Kulpa, extrait d'un roman de Xavier-Agnan Pommeret. Pour une étude détaillée de ce «spectacle-manifeste » et une écoute d'extraits d'archives sonores, consulter le livre-CD de Brigitte Joinnault, Antoine Vitez. La mise en scène des textes non dramatiques, Paris, Max Milo et L'Entretemps, 2019. Voir également, « Genèse 
d'un spectacle en forme de roman-photo ", in Brigitte Joinnault (dir.), Antoine Vitez, homme de théâtre et photographe, Besançon, Les Solitaires Intempestifs, 2015, p. 103-120.

21. Antoine Vitez, TDI, 475.

22. Anne Ubersfeld, Antoine Vitez, metteur en scène et poète, Montpellier, Éditions des Quatre Vents, 1987, p. 37.

23. Henri Meschonnic, « Vitez dans Phèdre joue le rythme », Europe, n 854-855, 2000, p. 156.

24. Antoine Vitez, TDI, 190.

25. Ibid., 194.

26. Voir sur ce point Antoine Vitez, homme de théâtre et photographe, op. cit., et Brigitte Joinnault et Marie Vitez, "Rencontres du théâtre et de la photographie dans l'œuvre d'Antoine Vitez ", à paraître in Photographie et arts de la scène, Julie Noirot et Cosimo Chiarelli (dir.), Focales, nº 3, 2019.

27. Antoine Vitez, «La prosodie avec une parenthèse sur les collectivités ", Francfort, 1980, document conservé à la Bibliothèque nationale de France (fonds Antoine Vitez).

28. Ibid.

29. Antoine Vitez, «Madeleine Marion », propos recueillis par Anne Laurent, Intervention, $\mathrm{n}^{\circ} 77$, juillet-sept. 1986, TDI, 50.

30. « $[\mathrm{L}] \mathrm{a}$ rencontre entre les deux hommes s'est faite, le courant est immédiatement passé. A. Vitez a tout d'abord choisi de lire des poèmes de Jean à Chaillot puis décidé de lire une grande partie de la pièce en 1985 et, compte tenu du succès de cette lecture, il a décidé de monter la pièce en son entier. Ce fut le début d'une grande aventure, A. Vitez était sensible à l'approche de Jean concernant le théâtre des commencements, ils ont beaucoup échangé à ce sujet. » Extrait d'un courrier adressé par Anne-Marie Métellus à Brigitte Joinnault en mai 2018, cité avec l'accord de l'auteur.

31. Le montage comportait des poèmes publiés, pour la plupart dans le recueil Au pipirite chantant (Paris, Maurice Nadeau et Robert Laffont, 1978) et quelques poèmes inédits, parfois même encore en travail (« Armstrong » notamment).

32. Jean Métellus, Au pipirite chantant, op. cit., p. 154 et 159.

33. Jean Métellus, Anacaona, Paris, Hatier International, "Monde noir», 1986, p. 46. Acte II, scène 1, Frère Buyl. La pagination renverra par la suite à cette première édition de 1986. Nous indiquons en chiffres romains le numéro de l'acte et en chiffres arabes celui de la scène. Par exemple, II, 1, 46.

34. II, 3, Frère Buyl, 52.

35. II, 3, Hodeja, gouverneur, 51.

36. La reine Anacaona, le roi Caonabo, Altabeira (à la fois confidente d'Anacoana et messagère des dieux), un serviteur de la reine, deux représentants du peuple (baptisés premier Indien et deuxième Indien), et Yaquimex (un résistant).

37. Frère Buyl (un missionnaire), Roldadilla (un juge), Hodeja et Ovando (deux gouverneurs).

38. III, 2, 93-94.

39. IV, 3, 139. Claude Mouchard explique qu'il s'agit du premier vers d'une poésie dont Métellus écrit dans Haïti, une nation pathétique (Paris, Denoël, 1987) qu'elle est habituellement attribuée à Anacaona, «symbole de la poésie taino ». Claude Mouchard, «Jean Métellus écrivain », Le Journal de Chaillot, $\mathrm{n}^{\circ}$ 37, janvier 1988, p. 126.

40. Transcription d'après l'enregistrement conservé à la BnF (fonds Antoine Vitez), ASPBAN_001343. Les indications en italique et entre parenthèses correspondent à des éléments non verbaux.

41. D’après les souvenirs d'Anne-Marie Métellus, Antoine Vitez avait apporté une pomme, une orange, une banane, et un chapeau. Le chapeau servait pour les Espagnols et les fruits pour les Indiens. On entend dans l'archive des objets dont la manipulation est « sonore ", mais on ne sait ni où était placé le micro ni en quoi était fait le plateau de la table.

42. ASPAN_001343_V1_1.A. 
43. Antoine Vitez, «L'ancien et le moderne », Journal de Chaillot, n 34, juin 1987, p. 14.

44. Ibid.

45. Ibid., p. 13.

46. ASPBAN_001341_V1_1.A et ASPBAN_001342_V1_1.A.

47. En novembre 1987, les élections sont annulées suite à de très graves violences. En janvier 1988, l'armée organise de nouvelles élections. Leslie Manigat est élu en février 1988 et renversé en juin par le général Namphy.

48. Le nom de David Recoing avait été accidentellement oublié dans le programme du spectacle, qui mentionnait seulement «musique de Georges Aperghis ». Cet oubli initial a perduré dans les publications ultérieures et dans les bases de données qui ont établi leurs notices à partir du programme.

49. Georges Aperghis a écrit Simata (pour piano ou clavecin préparé) en 1971, et «Cinq petits moments brefs » (pour clavecin) en 1988.

50. Antoine Vitez, "Anacaona », Journal de Chaillot, $n^{\circ}$ 34, juin 1987, p. 13, repris in Antoine Vitez, Écrits sur le théâtre, t. 4, Paris, P.O.L, 1997, p. 293.

51. Murray Grönwall jouait, en amateur, de l'alto. En 1968, dans sa mise en scène de La Grande Enquête de François-Félix Kulpa de Xavier-Agnan Pommeret, Antoine Vitez lui avait demandé d'être constamment en scène avec son instrument, de sorte qu'il jouât non seulement avec son corps mais aussi avec son alto. Antoine Vitez considérait que sa découverte de la possibilité de jouer avec la musique appréhendée comme un des possibles langages scéniques s'était précisément produite à ce moment-là. L'utilisation scénique de l'alto dans Anacaona créait, pour ceux qui en avaient été témoins, un rapprochement entre ces deux spectacles, ainsi qu'entre le missionnaire de la pièce de Métellus et le commissaire de celle de Pommeret.

52. Ce principe a d'abord été imaginé et mis en œuvre en 1974 pour la mise en scène des Miracles. Sur ce travail et les expérimentations souffle/chant/parole voir Brigitte Joinnault, «Mettre en scène La Bible, une expérience cardinale dans l'histoire du duo fraternel Antoine Vitez/Georges Aperghis ", in Guy Freixe et Bertrand Porot (dir.), Les Interactions entre musique et théâtre, Montpellier, L'Entretemps, 2011, p. 155-171.

53. Dans la première scène, par exemple, pour à peine plus de deux cents vers, on compte quarante-deux occurrences de noms propres indiens: vingt-et-une occurrences de noms de personnages (treize pour Guakanagarik, trois pour Guanikana, nom donné à Colomb par les Indiens en reconnaissance de la relation fraternelle qu'il avait apparemment établie avec Guakanagarik, trois pour Caonabo, deux pour Altabeira), neuf occurrences de noms de lieux ou de peuples (trois pour le Marien, deux pour le Xaragua, une pour la Maguana, l'île d'Ayay, la terre d'Ayti-Quisqueya-Bohio, les Arawaks), douze noms de divinités indiennes (deux pour Zémès, une pour Yocauna, Bayacou, et Cimi, sept pour Corocoté) auxquels s'ajoutent des noms communs tels les nytainos (les ancêtres), les butios (les devins), les karbets (des cases), le kohiba (une sorte de tabac), le cachiman (un fruit) ou la cassave (une galette).

54. II, 5, 64.

55. Quatre occurrences au premier acte, deux dans le récit du combat de Caonabo dans le Marien que fait le premier Indien (I, 6, 38-39), deux dans la réaction d'Anacaona après le récit $(\mathrm{I}, 6,41)$. Une occurrence au deuxième acte, cri de guerre de Caonabo (II, 2, 50). Treize occurrences au troisième acte : Yaquimex, deux fois, comme un chant de résistance, « Aya bombé, Aya bombé, Mourir plutôt qu'être asservis !» (III, 4, 102-103), Anacaona, neuf fois (III, 4, 104 à 110), tous les Indiens en chœur, deux fois (III, 4,110). Six occurrences au quatrième acte, dans la réplique finale de Yaquimex, à la fin de chaque strophe (IV, 3, 135 à 140).

56. « Aya bombé, Aya bombé

Le ciel a tonné

La mer a grondé

La terre a tremblé 
Le vent a mugi

Les montagnes ont frémi

L'Afrique est venue

Aya bombé, Aya bombé » (IV, 3, 140).

57. II, 1, 47. Indications de jeu d'après l'archive sonore ASPBAN_001341_V1_1. Les textes en italique correspondent aux ajouts d'Antoine Vitez.

58. Une spectatrice, propos transcrits.

59. Antoine Vitez, propos transcrits, ibid.

60. Jean Métellus, «Une parole haïtienne dans la langue française », Journal de Chaillot, $\mathrm{n}^{\circ} 37$, janvier 1988, p. 124-125.

61. Ibid.

62. Table ronde organisée par le Goethe Institut de Paris, saison 1981-82. Document BnF (fonds Antoine Vitez), ASBPAN_001602_V1_1.

63. Antoine Vitez, in Émile Copfermann, Conversations avec Antoine Vitez, op. cit, p. 152.

64. Daniel Soulier, "À propos de Bakary Sangaré ", dossier Conversations avec Antoine Vitez, 7 juillet 2003, Studio-Théâtre, archives de la Comédie-Française.

\section{RÉSUMÉS}

Cette contribution prend appui sur des archives sonores pour revenir sur le rôle essentiel de la diction dans le travail scénique d'Antoine Vitez et sur la métaphore éclairante de la "traduction généralisée » que Georges Banu et Danièle Sallenave ont présentée comme « la passion rectrice » de sa vie et de son œuvre. L'écoute des documents montre concrètement comment Vitez et les acteurs avec qui il travaillait mettaient en jeu la dimension sonore des textes, faisant de la scène, d'une part, un lieu privilégié de conservation et d'écoute de la diversité et de l'étrangeté du verbe, d'autre part, un lieu d'expérimentation de l'altérité dans le rapport aux langues, aux accents, aux voix. Apparaît une véritable philosophie de la relation et une manière de pratiquer concrètement, grâce au théâtre, un idéal relationnel. Nous accordons une attention particulière aux différentes présences de l'œuvre poétique et dramatique de Jean Métellus à Chaillot, et spécialement à la mise en scène d'Anacaona qui offre un point d'appui remarquable pour penser le rôle de la parole dans l'histoire politique et sociale, et terminons notre parcours dans les années 2000 par quelques extraits de la mise en scène des Conversations d'Émile Copfermann par Daniel Soulier dans lesquels Bakary Sangaré donne à entendre des propos d'Antoine Vitez sur l'importance de la langue comme seul bien que les hommes puissent partager sans fin.

This contribution relies on audio documents in order to reexamine the essential role of diction in Antoine Vitez's stage work and the illuminating metaphor of "generalized translation", which Georges Banu and Danièle Sallenave presented as "the governing passion" of his life and work. These documents testify specifically to how Vitez and the actors he worked with brought into play the sound dimension of texts, making the stage on one hand, a privileged space for listening to and preserving the diversity and the strangeness of the verb and, on the other, a space for experimenting with otherness in relation to languages, accents and voices. Here is a genuine relational philosophy and, thanks to theatre, a way to put a relational ideal into practice. We accord particular attention to the different performances of the poetic and dramatic works of Jean Métellus at Chaillot and, especially, to the staging of Anacaona, which offers a remarkable 
key element for conceiving the role of speech in political and social history. We end our journey in the early 21st century, with a few extracts from Émile Copfermann's Conversations with Antoine Vitez, staged by Daniel Soulier, in which Bakary Sangaré voices Vitez's comments on the importance of language as the only good that can be permanently shared.

\section{INDEX}

Mots-clés : écoute, diction, rapport entre les langues, langue nationale, langues étrangères, accents, voix, Vitez, Métellus, Anacaona

Keywords : listening, diction, relation between languages, national language, foreign languages, accents, voice(s), Vitez, Métellus, Anacaona

\section{AUTEUR}

\section{BRIGITTE JOINNAULT}

Brigitte Joinnault est maîtresse de conférences en études théâtrales à l'université de Nice-Sophia Antipolis, membre du Centre transdisciplinaire d'épistémologie de la littérature et des arts vivants et chercheuse associée à l'UMR THALIM. Ses recherches portent sur l'histoire et l'esthétique des écritures théâtrales des années 1945 à nos jours. Elle a réalisé de nombreux travaux sur les relations entre le théâtre et le non-théâtre (autres arts, sciences et réel). Elle conduit un projet de recherche sur les formes de présence de la photographie dans les écritures théâtrales (FoPPhET). Dans ce cadre, elle a réalisé en 2015 un livre-album collectif, Antoine Vitez, homme de théâtre et photographe, et elle prépare actuellement les actes du colloque international «La photographie au théâtre, XIX ${ }^{\mathrm{e}}$-XXI ${ }^{\mathrm{e}}$ siècle » organisé en novembre 2017 sous l'égide du laboratoire THALIM et du CTEL.

Parmi ses publications :

Antoine Vitez, homme de théâtre et photographe (dir.), Besançon, Les Solitaires Intempestifs, 2015. Relations théâtre/photographie. Tadeusz Kantor, mémoires imaginaires et filiations inconscientes, B. Joinnault et S. Fernandez (dir.), PHLIT, $\mathrm{n}^{\circ}$ 2, 2018 [en ligne]. http://phlit.org/press/? post_type $=$ articlerevue $\& \mathrm{p}=3150$ «Apparaissance de photographies de spectacles de Tadeusz Kantor dans Batailles et Rebatailles d'Alice Laloy ", in Relations théâtre/photographie. Tadeusz Kantor, mémoires imaginaires et filiations inconscientes, PHLIT, $\mathrm{n}^{\circ} 2,2018$ [en ligne].

« Photographie et audio-guides dans les dramaturgies de l'artiste québécois Philippe Ducros de Diapodiaspora à La Porte du non-retour ", in I. Ligier-Degauque et A. Teulade (dir.), La Mémoire de la blessure au théâtre. Mise en fiction et interrogation du traumatisme de la Renaissance au XXI ${ }^{e}$ siècle, Rennes, PUR, « Le spectaculaire », 2018, p. 23-37.

B. Joinnault et M. Vitez, « Rencontres du théâtre et de la photographie dans l'œuvre d'Antoine Vitez », in J. Noirot et C. Chiarelli (dir.), Photographie et arts de la scène, Focales, n 3, 2019, [en ligne], à paraître. 